\title{
ORDER LEVEL OPTIMIZATION IN INVENTORY MANAGEMENT USING ARENA SIMULATION MODEL
}

\author{
Milica Mitrović ${ }^{1}$, Dražen Popović ${ }^{2}$, Milorad Vidović ${ }^{3}$, Gordana Radivojević ${ }^{4}$ \\ 1,23,3 University of Belgrade, Faculty of Transport and Traffic Engineering, Vojvode Stepe 305, 11000 Belgrade, Serbia
}

Received 29 November 2020; accepted 25 January 2021

\begin{abstract}
By adequately managing inventories, companies adjust demand and supply over time to provide the necessary goods to meet customer requirements and make a profit. A simulation is a great tool for optimizing inventory management by examining system behavior in a variety of settings. This paper presents a simulation model developed in ARENA software to optimize inventory management, i.e. to determine the level of inventory at which it is necessary to order goods for which the system generates minimal total costs.
\end{abstract}

Keywords: simulation, optimization, inventory management, ARENA.

\section{Introduction}

Business modeling simplifies the representation of a real-life complex system to analyze significant measures of system performance. Such a simplified representation is called a model. The model represents the behavior of the modeled system based on which the creator of the model acquires knowledge and insight into the behavior of the system. The purpose of the simulation is to create an experimental model that represents the behavior of a real system in a certain period of time avoiding time and cost losses for optimal planning (Altiok et al., 2007). The simulation is widely applied in the fields of engineering, business, natural and social sciences. The simulation methodology relies on computer technology, statistics, and operational research (Bratley et al., 1987).

The simulation model is created in a computer program and is used as an alternative to analytical modeling. The difference between analytical and simulation modeling is their nature of the solution. The analytical model requires solving a mathematical problem, deriving mathematical formulas, and an algorithmic procedure to obtain significant measures of performance. The simulation model requires the launch of a simulation program based on the calculation of statistical parameters based on historical data to form significant performance measures (Altiok et al., 2007). Simulation software tools such as Arena, FlexSim, Extend, and Simio facilitate the development of computer simulation models, their use and provide the ability to visually monitor the flow of the simulated process. The application of simulation implies several activities that correspond to the phases of system analysis (Vidović, 2020):

- Problem formulation;

- Development of a conceptual model of the system;

${ }^{1}$ Corresponding author: milica.mitrovic@sf.bg.ac.rs 
- Computer program development;

- Model testing and verification;

- Realization of simulation experiment; and

- Interpretation of results.

Conceptual modeling implies the creation of a simplified model of a real system and represents the most important phase of simulation modeling. The creation of the model affects all phases of modeling related to data collection, the speed of model development and experiment implementation, the accuracy of the model, and the confidence placed in the output results. The good quality of model creation is a condition for successful simulation modeling. Conceptual modeling refers to the observation of real system problems that need to be solved using a simulation model. The simulation process of the model is constantly repeated through iterations in the life cycle of the simulation study. Therefore, conceptual modeling is not a one-time process but is repeated and refined several times during the simulation. The four key processes in developing and using a simulation model are conceptual modeling, model coding, experimentation, and implementation. The result of each process is a conceptual model, a computer model, a solution to a problem, and a better understanding of the real world (Robinson, 2008).

A conceptual model represents a phase in a simulation model that determines the components and structures of a system's simulation model. The model creator must understand the exact problem and object of the simulation and turn it into a suitable simulation model. The conceptual model consists of four main components: determining modeling goals, identifying inputs to the model, defining outputs from the model, and defining the content of the model. There are two types of determining modeling goals. The first type of objective refers to the description of the purpose of the model and project modeling. The second type refers to the general objectives of the project which are related to the time parameter of the project, the nature of the model, the speed of work, the visual display, and the ease of use of the model. Different modeling goals lead to different models and essentially describe the conceptual model. The identified inputs of the model are elements that can be changed during the simulation to improve and better understand the problems of the real system according to certain goals. The defined outputs from the model represent the results of the simulation model reporting based on which it is determined whether the modeling goals have been achieved and if not, they indicate the reason why the goals are not achieved. Defining the content of the model consists of the components in the model and their interrelationships. Conceptual modeling is the process of creating a conceptual model that requires the following activities (Robinson, 2008):

- Understanding the problems of the real system;

- Determining the model and general objectives of the simulation;

- Defining the output of the model;

- Identification of model inputs;

- Determining the content of the model (scope and level of detail), identifying assumptions and simplifications.

The aim of this paper is to determine the level of inventory at which order is performed with the lowest total costs, using a simulation model developed in ARENA (Rockwell Automation) software. Total costs are 
affected by the cost of ordering, the cost of owning stock, and the lack of stock. The purpose of this model is to monitor the behavior of a real inventory system over time to determine the level of inventory with the lowest total cost at which order is performed and which meets customer requirements.

The concept of the work is organized into three chapters. The second chapter describes inventory management, consumption characteristics, and which inventory management strategy is used for this simulation model. The third chapter describes the simulation model, i.e. the parameters of the model and the way of developing the model. Finally, the output results of the simulation model and the concluding considerations are presented.

\section{Inventory Management}

The logistics process of the inventory system works optimally if, with a minimum of working capital and the use of the necessary fixed assets, workforce, and energy, the possibility of maximum profit from the formed inventory is provided. To determine the desired state of inventories (structure and quantity), it is necessary to know the way of functioning of the business system in which the problems of stocks are investigated. Inventory management is influenced by factors, i.e. techno-economic characteristics that are of the greatest importance for the functioning of the inventory system. Technoeconomic characteristics that are important for determining the state of inventories are (Vukićević, 1995): types of inventories and their structure, physical and chemical properties of inventories, a system of supply and consumption of inventories, inventories replenishment, a function of costs incurred by inventories, a strategy of costs incurred by the stock-out, as inventory management strategy, control system, and restrictions.

Based on the importance of consumption, stocks are formed to respond to market demands according to the indicated need and demand. In different business systems, consumption can have different characteristics that can be (Vukićević, 1995):

- Continuous or discontinuous;

- Constant or variable;

- Deterministic or stochastic;

- Stationary or non-stationary;

- With or without restriction;

- Dependent or independent in relation to the consumption of other products, seasonal character, days of the week, etc.

Inventories are managed according to a predefined strategy. The inventory management strategy depends on other techno-economic characteristics that are present in the business system. An important factor in choosing a strategy is the choice of sizes through which inventories are managed. In the business system, the most common inventory management strategies are (Vukićević, 1995):

- $\quad\left(q^{*}, r_{n}^{*}\right)$ - quantity to be procured and stock level at which it is ordered;

- $\left(q^{*}, W^{*}\right)$ - quantity procured and time between two adjacent procurements;

- $\quad\left(M^{*}, r_{n}^{*}\right)$ - maximum allowed quantity of stock and level of stock at which it is ordered;

- $\quad\left(M^{*}, W^{*}\right)$ - maximum allowed quantity of stock and time between two adjacent procurements;

- $\quad\left(q^{*}, Y\right)$ - quantity procured with known delivery time $(Y)$ for statistical models;

- $\quad\left(q^{*}, R_{n}^{*}, W_{p}^{*}\right),\left(M^{*}, R_{n}^{*}, W_{p}^{*}\right)$ - where the quantity $\left(W_{p}^{*}\right)$ represents the time between two adjacent stock checks (periodic check system). 
In this paper, the strategy $\left(q^{*}, R_{n}^{*}, W_{p}^{*}\right),\left(M^{*}, R_{n}^{*}, W_{p}^{*}\right)$ is used for the simulation model inventory management described by four parameters:

- $q^{*}$ - quantity to be procured;

- $R_{n}^{*}$ - a stock level at which it is ordered;

- $W_{p}^{*}$ - time between two adjacent stock checks; and

- $M^{*}$ - maximum allowed amount of stock.

The strategy $\left(q^{*}, R_{n}^{*}, W_{p}^{*}\right),\left(M^{*}, R_{n}^{*}, W_{p}^{*}\right)$ occurs in situations when the buyer purchases goods with a defined delivery time and has a stochastic consumption of goods and a fixed storage capacity, where it is important that the goods always fill the warehouse. The main problem with this strategy is that the buyer knows the quantity of goods that he can accept only at the moment when the goods are delivered to him. In this case, the agreed quantity represents the approximate quantity of delivery, which creates certain difficulties for the supplier in planning the delivery. With this strategy, the buyer may run out of stock for some time during the delivery period (Vukićević, 1995).

\section{Simulation Model}

This paper uses the ARENA software tool to create a simulation model of inventory management of an item in which stocks are checked daily and in case they are less than the safety level, an order is performed from the supplier. The demand for an item (exit from the warehouse) is a stochastic quantity and is generated at the day level in the pallets. From the moment of ordering to the moment of arrival of the goods in the warehouse, a few days pass. Backordering is possible in the system, i.e. if on a given day there is not enough stock in stock to meet the output, these requests can be served in the following period upon arrival of goods from the supplier (system stock may have a negative value in this case which incurs additional costs).

The selected model parameters that affect the creation of the simulation are described below. Daily demand is defined with a normal distribution ( $\mu^{\text {output }}=10, \sigma^{\text {output }}=2.5$ ) in the pallets. The maximum stock level to which the order quantity is calculated is $Q_{\max }=150$ pallets. The time required for the goods to arrive from the supplier after the order is placed is defined with a normal distribution ( $\mu^{\text {time }}=3, \sigma^{\text {time }}=0.5$ ) in days. Ordering goods from suppliers is done for cases when the stock level falls below safety level $Q_{\min }$ and this value is the goal of the simulation optimization. Different values of this parameter were observed in the simulation runs and we have presented in this paper the results for a selected range of values (values that are close to the best solution) $Q_{\min }: 20,25,26,27,28,29,30,31$, $32,33,34,35,40$ and 50 pallets.

The quantity of the order $(Q)$ depends on the current stock status (S), maximum stock level $Q_{\max }$, expected delivery time ( $\left.\mu^{\text {time }}\right)$, and expected demand ( $\left.\mu^{\text {output }}\right)$ :

$Q=A N I N T\left(Q_{\max }+\mu^{\text {time }} \cdot \mu^{\text {output }}-S\right)$

Where ANINT represents a command in ARENA software through which numbers are rounded to integer values (only whole pallets of the product can be ordered).

When ordering goods is generated, additional orders are not possible until the order is delivered, i.e. only when the ordered goods arrive, is it possible to generate the next order (otherwise there would be multiple active orders, one per each day until the first order has arrived). The initial stock state at the beginning of the simulation is 
$S=100$ pallets. The state of stocks can be positive or negative (negative in the case that customers are looking for goods and the same is not in stock, in which case the service is postponed for the next day when there are enough goods in stock). Additionally, two more stock related variables are used in the simulation model, for calculation of inventory cost $s^{\text {stock }}$ (cost of storage and handling) and for stockout cost $S^{\text {deficit stock }}$ (cost of low service to the customer). The state of stock is systematically monitored $\left(S^{\text {stock }}\right)$, i.e. when the stock is "positive" $S \geq 0$, the cost of stock on hand is calculated. The variable $S^{\text {stock }}$ takes value 0 when inventories are in deficit. When the stock is "negative" $S<0$ the cost of the deficit stock is calculated as the absolute value, and the variable $S^{\text {deficit stock }}$ takes value 0 when inventories are in the plus. The daily cost of having inventory per pallet is $C^{\text {stock }}=0.1 €$, the daily cost of running out of inventory per pallet is $C^{\text {stock deficit }}=3 €$, and the cost of the order is $C^{\text {order }}=5 €$. The simulation observes the total costs for 5 and 10 replications and for replication lengths of $365,10 * 365,20 * 365$, and $50 * 365$ days (8 variants of different lengths and number of replications for the purpose of sensitivity analysis).

Two parallel submodels are created for this simulation. The first submodel refers to the daily checking of the situation and ordering of stocks where the entity is the procurement, i.e. pallets input into the warehouse. The second submodel refers to delivery where the entity is consumption, i.e. pallets output from the warehouse. The number of orders, the positive state of stocks, and the lack of stocks are systematically monitored via three different variables. Figure 1 shows a simulation model of inventory management and a visual representation of the movement of inventory levels over time.

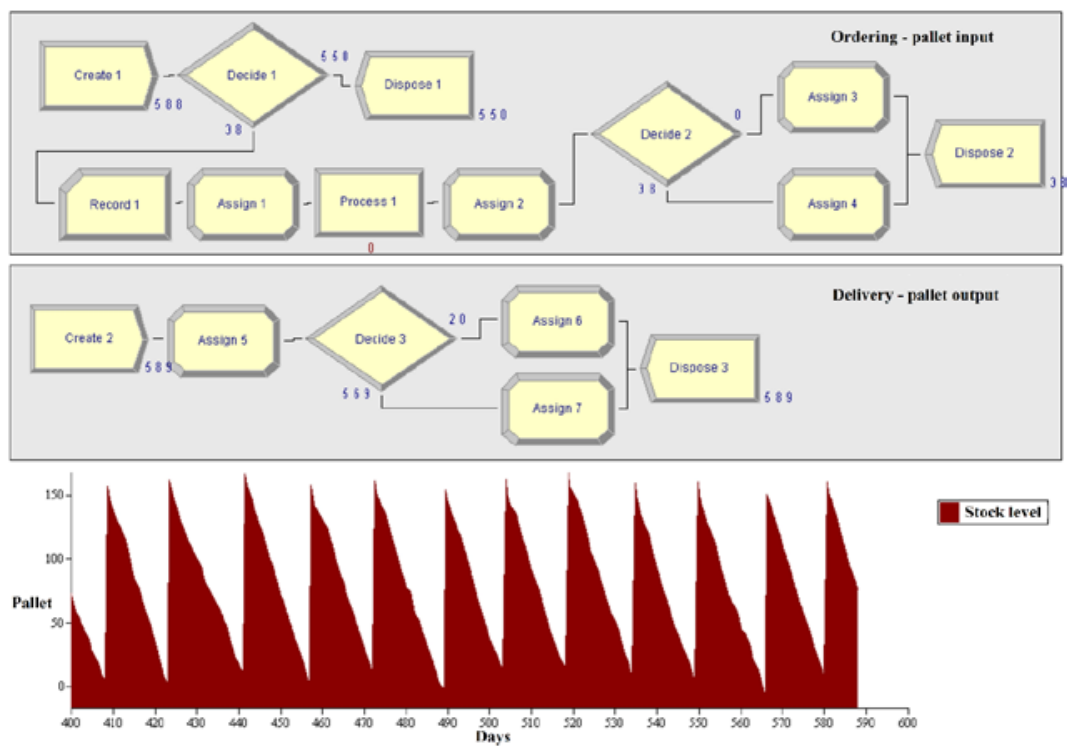

Fig. 1.

ARENA Simulation Model of Inventory Management with Inventory Level Plot over Time for One Case of Simulation Parameters 
In the ordering submodel (pallet input), the Create 1 module defines the entry of entities into the system. The next step is the Decide 1 module in which it is checked whether it is necessary to generate the ordering of goods from the supplier (if there is no order on the way and if the stocks are below the minimum level $Q_{\min }$, ordering is done). The need for order generation is made once each day (entity is generated constantly 1 per day in the Create 1). The Record 1 module records the order number which is required to determine the ordering costs. In the Assign 1 module, the order quantity $Q$ is determined (by equation 1). Then follows the Process 1 module, which simulates the transport of goods (order lead time to arrive at the warehouse from the moment of placement the order) defined by the normal distribution ( $\mu^{\text {time }}=3, \sigma^{\text {time }}=0.5$ ) in days. The Assign 2 module records the stock level $S$ (it increases the variable $S$ for the quantity of ordered goods). The Decide 2 module is then added to separate the flows for systemic monitoring of $S^{\text {stock }}$ and $s^{\text {deficit stock }}$ . If the stock variable $S$ is less than 0 True branch represents the Assign 3 module that makes changes to two variables $\left(S^{\text {stock }}\right.$ and $\left.S^{\text {deficit stock }}\right)$, in which case the $S^{\text {stock }}$ is equal to 0 , and the $S^{\text {deficit stock }}$ records a positive value equivalent to the absolute value of $S$. The False branch represents the Assign 4 module, which also records the above two variables, in which case the $S^{\text {deficit stock }}$ is equal to 0 , and the $S^{\text {stock }}$ takes value $S$. In both cases, the entity leaves the system with the Dispose 2 module.

In the delivery submodel (pallet output), the Create 2 module defines the arrival of demand from customers, i.e. pallet output from the system. This demand entity is generated once per day. The quantity of demand is defined in the following Assign 5 module where stock variable $S$ is stochastically reduced with the normal distribution ( $\mu^{\text {output }}=10, \sigma^{\text {output }}=2.5$ )
. The Decide 3 module is used to separate the flows of positive stocks and shortage of stocks. The Decide 3 module checks if the $S<0$. In the case of True outcome, the entity goes to Assign 6 module where $S^{\text {stock }}$ takes value 0 , and at the same time, the $S^{\text {deficit stock }}$ takes the absolute value of the $S$ variable. The False branch of the Decide 3 goes to the Assign 7 module where the $S^{\text {deficit stock }}$ is equal to 0 and the $S^{\text {stock }}$ is equal to the current state of $S$ variable. In both cases, the entity leaves the system with the Dispose 3 module.

After all the previously described steps of the model have been realized, the necessary settings should be made in the Run Setup form (simulation run parameters regarding the number and length of replications). After the completion of the last replication in Reports /User Specified, the observed parameters are observed, which are: the average number of orders, the average state of positive stocks, and the average state of a shortage of stocks. Based on these parameters, the total costs are determined by different levels of stocks when ordering and replication lengths.

\section{Results}

Table 1 shows the total costs at the annual level for different variants of ordering levels $\left(Q_{\min }\right)$, number, and length of simulation replication. Simulations of 5 and 10 replications for replication lengths of 365 , $10 * 365,20 * 365$, and $50 * 365$ days were observed in the model in order to analyze the influence of simulation intensity and simulation time length on the solution. Based on the average values of the parameters, the total costs of the observed simulation variants are determined, which are comparatively analyzed and used to decide which stock order level $\left(Q_{\min }\right)$ is the best. Comparative analysis of the influence of the 
number of replications on the solution that generates the lowest total costs indicates a great similarity of the obtained results with a small deviation in the period of 365 and $20^{*} 365$ days. As for the length of the simulation time, at a length of $50 * 365$ days, the number of replications does not affect the solution $Q_{\min }$ at which the system generates minimal costs. Table 1 shows the results for stock levels at which 25 to 35 pallets are ordered with step 1 (this range includes the best solutions for 8 variants of the number and length of replications), as well as for the two extremes related to the low stock level of 20 pallets and an extremely high stock level of 50 pallets to compare their costs. Extremely low stocks generate lower costs of ordering and stock on hand and higher costs of lack of stocks, while extremely high stocks have the opposite effect. In both cases, the total cost is significantly higher than a solution in which there is a good balance of $Q_{\min }$ level regarding the excessiveness or lack of stock, as well as the frequency of ordering. Also, it should be noted that the cost incurred by stock-out (Z-and TZ- shown in Tables 2 and 3) is the most important one to reduce due to the importance of maintaining the high service level to the customers' demands.

In the case of 5 replications, the total cost is the lowest at the level of $Q_{\min }=33$ pallets, while in the case of 10 replications the costs are the lowest at the level of $Q_{\min }=30$ pallets for the replication length of 365 days. With a replication length of $20^{*} 365$, the deviation is smaller, for the case of 5 replications the lowest costs are at the level of $Q_{\min }=31$ pallets, and in the case of 10 replications, they are the lowest at the level of $Q_{\min }=32$ pallets. The absolute coincidence of the obtained results of the level at which ordering is performed for both replications is for the replication length of $10^{*} 365$ and $50 * 365$, which is the level of $Q_{\min }=31$ pallets. Small deviations in the solutions are a consequence of the stochasticity of the process and the insufficient length and intensity of the simulation in variants with a smaller number of replications and a shorter simulation time.

\section{Table 1}

Total Annual Costs for different Variants of $Q_{\min }$ Number and Length of Simulation Replications

\begin{tabular}{|c|c|c|c|c|c|c|c|c|c|c|c|c|c|c|c|}
\hline \multirow{2}{*}{ 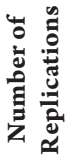 } & \multirow{2}{*}{ 总泀 } & \multicolumn{14}{|c|}{$Q \min$} \\
\hline & & 20 & 25 & 26 & 27 & 28 & 29 & 30 & 31 & 32 & 33 & 34 & 35 & 40 & 50 \\
\hline \multirow{4}{*}{5} & 365 & 3407 & 3145 & 3114 & 3109 & 3114 & 3090 & 3067 & 3070 & 3057 & 3056 & 3062 & 3082 & 3153 & 3339 \\
\hline & $10^{*} 365$ & 3307 & 3131 & 3102 & 3098 & 3075 & 3068 & 3072 & 3075 & 3068 & 3076 & 3090 & 3093 & 3162 & 3332 \\
\hline & $20 * 365$ & 3298 & 3124 & 3099 & 3096 & 3072 & 3067 & 3070 & 3066 & 3067 & 3074 & 3084 & 3085 & 3155 & 3333 \\
\hline & $50 * 365$ & 3302 & 3127 & 3103 & 3091 & 3077 & 3068 & 3067 & 3064 & 3067 & 3070 & 3078 & 3083 & 3149 & 3329 \\
\hline \multirow{4}{*}{10} & 365 & 3361 & 3129 & 3100 & 3100 & 3092 & 3074 & 3060 & 3061 & 3063 & 3061 & 3068 & 3073 & 3159 & 3335 \\
\hline & $10^{*} 365$ & 3307 & 3131 & 3099 & 3094 & 3072 & 3070 & 3069 & 3067 & 3063 & 3074 & 3080 & 3086 & 3162 & 3331 \\
\hline & $20 * 365$ & 3307 & 3128 & 3100 & 3091 & 3071 & 3068 & 3070 & 3065 & 3063 & 3074 & 3078 & 3083 & 3156 & 3333 \\
\hline & $50 * 365$ & 3306 & 3127 & 3102 & 3090 & 3075 & 3070 & 3067 & 3064 & 3065 & 3071 & 3076 & 3082 & 3151 & 3331 \\
\hline
\end{tabular}


Figure 2 presents a graphical comparison of the total costs of 5 and 10 replications by replication lengths, i.e. for $365,10 * 365$, $20 * 365$, and $50 * 365$ days. Figure 2 shows an increasing match of total costs with a longer replication for both simulated replications. At a replication length of 365 days, a small deviation is observed, while this deviation is less and less with longer length of replications. With the last replication length of $50^{*} 365$ days, the result of the total costs for both replications is almost the same.

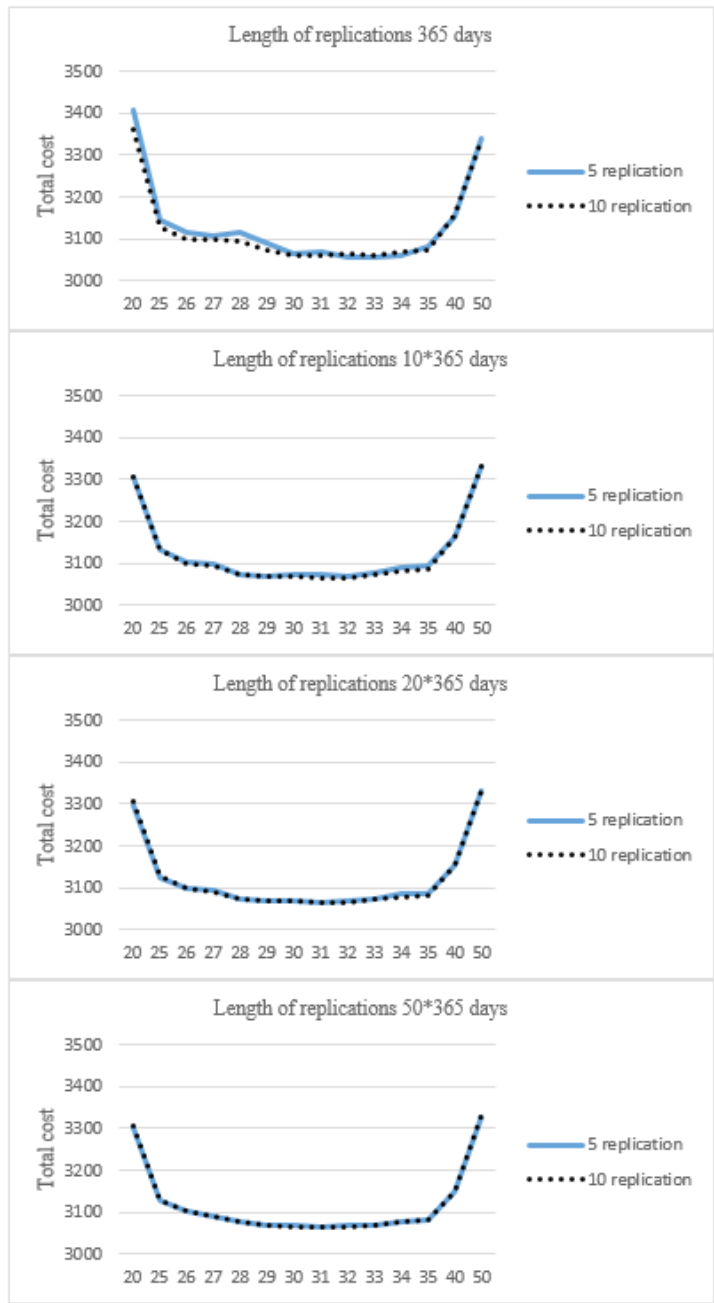

Fig. 2.

Comparison of the Total Cost of the Number of Replications according to the Number and Length of Replications 
Tables 2 and 3 show the simulation output parameters for two cases of replication length (365 days and 50*365 days) and the number of replications ( 5 and 10). Table 3 show the annual values of parameters (average yearly values). The output parameters of the simulation are the average number of orders $(P)$, positive stock state $(\mathrm{Z}+)$, and stock shortage state (Z-), based on which ordering costs (TP), stock ownership costs $(\mathrm{TZ}+)$, stock shortage costs (TZ-) and total costs $(\Sigma \mathrm{T})$. Total inventory costs represent the sum of the cost of ordering, the cost of owning inventory, and the cost of a shortage of inventory. The average number of orders (average of the total number of orders) and possession of stocks (daily average) increases with a higher level of stocks, which directly refers to their costs. While the average number of stock shortages (daily average) and its costs decrease with a higher level of stock at which the order is made. Based on the obtained results for different variants of stock levels when ordering, it is noticed that there is a small deviation of the total costs between these two simulation variants (two extremes), where the simulation variant with extreme long replication of 50 years and 10 replications favorizes the reduction of stock-out costs which is more important than having somewhat increased costs of stocks on hand.

Table 2 shows the simulation output parameters for different ordering levels based on which the total costs for the replication length of 365 days and 5 replications were calculated. The lowest total costs for this case are at the level of 33 pallets. Table 3 also shows the simulation output parameters for different ordering levels based on which the total costs were calculated, but for a replication length of $50 * 365$ days and 10 replications. The lowest total costs for this second case are at the level of 31 pallets. The performed analysis of the impact of simulation intensity shows lower costs for longer simulation time, i.e. the obtained solutions show that the costs are lower for the case of 50*365 days and 10 replications at the level of 31 pallets, compared to the first simulation of 365 days and 5 replications at the level of 33 pallets.

Table 2

Output Parameters for Replication Length of 365 Days and 5 Replications

\begin{tabular}{|c|c|c|c|c|c|c|c|c|c|c|c|c|c|c|}
\hline Qmin & $\mathbf{2 0}$ & $\mathbf{2 5}$ & $\mathbf{2 6}$ & $\mathbf{2 7}$ & $\mathbf{2 8}$ & $\mathbf{2 9}$ & $\mathbf{3 0}$ & $\mathbf{3 1}$ & $\mathbf{3 2}$ & $\mathbf{3 3}$ & $\mathbf{3 4}$ & $\mathbf{3 5}$ & $\mathbf{4 0}$ & $\mathbf{5 0}$ \\
\hline $\mathrm{P}$ & 22.00 & 22.80 & 23.00 & 23.00 & 23.00 & 23.20 & 23.20 & 23.60 & 23.80 & 24.00 & 24.20 & 24.40 & 25.00 & 26.80 \\
\hline $\mathrm{Z}+$ & 72.52 & 74.83 & 75.76 & 76.31 & 76.70 & 77.03 & 77.11 & 77.32 & 78.50 & 78.83 & 79.04 & 79.63 & 82.19 & 87.80 \\
\hline $\mathrm{Z}-$ & 0.594 & 0.273 & 0.214 & 0.190 & 0.182 & 0.148 & 0.124 & 0.119 & 0.067 & 0.053 & 0.051 & 0.049 & 0.025 & 0.000 \\
\hline $\mathrm{TP}$ & 110.0 & 114.0 & 115.0 & 115.0 & 115.0 & 116.0 & 116.0 & 118.0 & 119.0 & 120.0 & 121.0 & 122.0 & 125.0 & 134.0 \\
\hline $\mathrm{TZ}+$ & 2646.9 & 2731.2 & 2765.4 & 2785.5 & 2799.5 & 2811.6 & 2814.5 & 2822.0 & 2865.2 & 2877.3 & 2884.9 & 2906.6 & 3000.1 & 3204.8 \\
\hline $\mathrm{TZ}-$ & 650.3 & 299.4 & 233.9 & 208.4 & 199.5 & 162.5 & 136.2 & 130.1 & 73.2 & 58.4 & 55.8 & 53.7 & 27.5 & 0.5 \\
\hline $\mathrm{ST}$ & 3407.2 & 3144.5 & 3114.3 & 3108.9 & 3114.0 & 3090.1 & 3066.7 & 3070.1 & 3057.4 & $\mathbf{3 0 5 5 . 7}$ & 3061.8 & 3082.3 & 3152.6 & 3339.3 \\
\hline
\end{tabular}




\section{Table 3}

Output Parameters for Replication Length of 50*365 Days and 10 Replications

\begin{tabular}{|c|c|c|c|c|c|c|c|c|c|c|c|c|c|c|}
\hline Qmin & $\mathbf{2 0}$ & $\mathbf{2 5}$ & $\mathbf{2 6}$ & $\mathbf{2 7}$ & $\mathbf{2 8}$ & $\mathbf{2 9}$ & $\mathbf{3 0}$ & $\mathbf{3 1}$ & $\mathbf{3 2}$ & $\mathbf{3 3}$ & $\mathbf{3 4}$ & $\mathbf{3 5}$ & $\mathbf{4 0}$ & $\mathbf{5 0}$ \\
\hline $\mathrm{P}$ & 22.09 & 22.77 & 22.93 & 23.07 & 23.21 & 23.36 & 23.53 & 23.67 & 23.83 & 23.98 & 24.13 & 24.29 & 25.13 & 26.99 \\
\hline $\mathrm{Z}+$ & 1.46 & 1.51 & 1.52 & 1.53 & 1.53 & 1.54 & 1.55 & 1.56 & 1.57 & 1.58 & 1.59 & 1.60 & 1.65 & 1.75 \\
\hline $\mathrm{Z}-$ & 0.010 & 0.005 & 0.004 & 0.003 & 0.003 & 0.002 & 0.002 & 0.002 & 0.001 & 0.001 & 0.001 & 0.001 & 0.000 & 0.000 \\
\hline $\mathrm{TP}$ & 110.4 & 113.9 & 114.7 & 115.4 & 116.1 & 116.8 & 117.6 & 118.4 & 119.1 & 119.9 & 120.7 & 121.5 & 125.7 & 135.0 \\
\hline $\mathrm{TZ}+$ & 2664.4 & 2751.4 & 2765.6 & 2786.6 & 2799.2 & 2816.4 & 2836.8 & 2855.1 & 2872.3 & 2891.2 & 2906.2 & 2920.8 & 3012.8 & 3196.2 \\
\hline $\mathrm{TZ}-$ & 530.9 & 261.6 & 221.8 & 187.9 & 160.2 & 136.5 & 112.2 & 90.5 & 74.0 & 59.9 & 48.8 & 40.1 & 12.1 & 0.3 \\
\hline$\Sigma \mathrm{T}$ & 3305.7 & 3126.9 & 3102.1 & 3089.9 & 3075.4 & 3069.8 & 3066.6 & $\mathbf{3 0 6 4 . 0}$ & 3065.4 & 3071.0 & 3075.7 & 3082.3 & 3150.6 & 3331.4 \\
\hline
\end{tabular}

\section{Conclusions}

In this paper, using the simulation software Arena, a simulation model was developed for determining the level of stock in which the ordering of goods with the lowest total costs is performed. By applying the presented simulation model, one can gain insight into the behavior of the inventory management system, i.e. the impact of changing the business decision on the minimum inventory level at which goods are ordered from suppliers on individual costs of inventory, lack of inventory, and ordering. Based on the obtained simulation results, the stock level of 31 pallets realizes the lowest total costs for the observed problem setting. In future research, the simulation model can be extended for more detailed and complex observation of the real inventory management system (higher number of items, additional costs, failures in the system by customers who do not want to wait for goods and resources in the system, etc.).

\section{Acknowledgement}

This work is funded by the Ministry of Education, Science and Technological Development of the Republic of Serbia within the project TR 36006 for the period 2011-2020.

\section{References}

Altiok, T.; Melamed, B. 2007. Simulation Modeling and Analysis with ARENA. Elsevier, 435p.

Bratley, P.; Fox, L.B.; Schrage, E.L. 1987. A Guide to Simulation. Springer Science and Business Media, New York. 393p.

Robinson, S. 2008. Conceptual modelling for simulation Part I: definition and requirements, Journal of the Operational Research Society 59(3): 278-290.

Vidović, M. 2020. Lecture material from the course Simulations of logistics systems. University of Belgrade Faculty of Transportation and Traffic Engineering, Belgrade, Serbia.

Vukićević, S. 1995. Warehouses [In Serbian: Skladišta]. University of Belgrade - Faculty of Transportation and Traffic Engineering, Belgrade, Serbia. 652 p. 